\title{
Un proyecto de refundación democrática: el alfonsinismo frente al avance del mercado ${ }^{1}$
}

\author{
A project of democratic re-foundation: Alfonsinism facing the market advance
}

\author{
IgNACIO ANDRÉs Rossi \\ Universidad Nacional de General Sarmiento, Argentina
}

\begin{abstract}
RESUMEN La transición a la democracia en la Argentina formó parte de un proceso político atravesado por amplias transformaciones a nivel macroeconómico nacional, regional y mundial. El gobierno de Raúl Alfonsín (1983-1989) tuvo, luego de una salida democrática sin condicionantes formales, amplios márgenes para comenzar a configurar un régimen político democrático que, con el devenir del triunfo electoral y el apoyo de la ciudadanía, le permitió definir un programa político ambicioso. Este ensayo pretende analizar cómo, desde 1983 el alfonsinismo se propuso construir dicho programa, sus principales dificultades, el espíritu del mismo y algunas reflexiones en torno a los planes económicos y a la reforma del Estado que se sucedieron en aquel entonces. Así, en una primera parte se analiza el contexto macroeconómico mundial y latinoamericano, como de su impacto nacional en la transición argentina. Luego, se delimitan algunas precisiones sobre el comienzo de la transición a la democracia y el espíritu republicano que el alfonsinismo le imprimió a esta, reflexionando, a su vez, sobre sus propuestas de política económica frente al avance del neoliberalismo internacional.
\end{abstract}

PALABRAS CLAVE Transición democrática; proyecto refundacional; contexto económico; reformas.

\footnotetext{
1. Este trabo fue realizado en el marco del proyecto de investigación "Las interpretaciones heterodoxas en la historia económica argentina reciente" dirigido por la Dra. Forcinito Karina en el Área de Economía del Instituto del Desarrollo Humano de la Universidad Nacional de General Sarmiento (2018-2020).
} 
ABSTRACT The transition to democracy in Argentina was part of a political process that went through extensive transformations at the national, regional and global macroeconomic levels. After a democratic exit without formal conditions, the government of Raúl Alfonsín (1983-1989) had wide margins to begin to configure a democratic political regime that, with the evolution of the electoral victory and the support of the citizens, allowed it to define an ambitious political program. This essay aims to analyze how, since 1983, Alfonsinism proposed to build such a program, its main difficulties, its spirit and some reflections on the economic plans and the reform of the State that followed one another at that time. Thus, the first part analyzes the global and Latin American macroeconomic context, as well as its national impact on the Argentine transition. Then, some details about the beginning of the transition to democracy and the republican spirit that Alfonsinism imprinted on it are delimited, reflecting on their economic policy proposals against the advance of international neoliberalism.

KEY WORDS Democratic transition; refundational project; economic context; reforms.

\section{Introducción}

El presente ensayo de revisión bibliográfica pretende delimitar las propuestas y alcances de lo que entendemos como un proyecto de refundación democrática enmarcado en el gobierno de Raúl Alfonsín (1983-1989). Entendemos que el mismo, se desarrolló en un cuadro de profundas transformaciones internacionales y nacionales durante la década de los años ochenta, especialmente en el área de la macroeconomía y la política económica. El énfasis refundacional del alfonsinismo fue señalado por varios autores (Aboy Carlés, 2001; Barros, 2002; Zoppi Fontana, 1993), y como dicen Vitale y Dagatti (2016) desde su perspectiva, puede ser entendido como una serie de estrategias gubernamentales que desde el plano discursivo buscaba definir una frontera política entre un pasado indeseado y un provenir anhelado, a su vez, construido sobre un presente entendido como la base de las acciones que se implementarían para llegar al mismo. Desde este punto de vista, nos centramos en aquellas estrategias gubernamentales que con el fin de delimitar un proyecto político integral se enmarcaron en un cuadro de rediseño del régimen democrático con una intención refundacional propia del alfonsinismo y su partido la Unión Cívica Radical (UCR). La democracia alfonsinista fue un proceso sociopolítico que se tornó en un cuadro de amplios condicionamientos macroeconómicos mundiales dadas las transformaciones que el capitalismo atravesaba en aquellos años y que marcaron a Latinoamérica por la crisis 
de la deuda y los shocks externos negativos. Creemos que analizar el contexto internacional, regional y el contexto local argentino nos permitirá un mejor diálogo entre los condicionamientos externos de carácter económico y la política nacional de la transición a la democracia.

Las transformaciones de los Estados se tornaban como una empresa central en un contexto en el que el avance del neoliberalismo mundial promovía con más énfasis la soberanía del mundo de los negocios mediante la desregulación de los mercados. En este contexto, el alfonsinismo apelaba a consignas democráticas en el plano político, tales como la defensa de los derechos humanos, la libertad, el respeto por las disidencias y el pluralismo (Palermo, 2004) como forma de aglutinar a la sociedad en un proyecto de transición. Así, la UCR se asumía como el partido garante de las libertades republicanas y liberales, en combinación con reivindicaciones populares y de bienestar social que eran disputadas al partido tradicional justicialista (PJ). Sin embargo, posteriormente se debería enfrentar el contrapeso de una dinámica mundial que priorizaba los ajustes fiscales atentando contra el prometido bienestar social de la democracia (Ramírez, 2019). El fracaso económico del primer equipo de Alfonsín, al no lograr principalmente acuerdos de refinanciación de la deuda externa (Brenta, 2019) como de cierta estabilización de la inflación, los salarios reales y el déficit fiscal (Pesce, 2004), fue quizás el momento más nítido de que la influencia del capitalismo global canalizado en los organismos financieros internacionales y los acreedores externos no serían fácilmente eludibles. Fue allí cuando, sin abandonar jamás el ethos democrático que lo caracterizó desde 1983, el gobierno presentara un plan antinflacionario, conocido por la prensa como Plan Austral, pero denominado formalmente Plan de Reforma Económica, que constituyera una ambivalencia semejante al contraponer el reformismo modernizante del Alfonsín con el ortodoxo afín al neoliberalismo internacional. Los límites y fracasos del proyecto alfonsinista quizás puedan entenderse con la lectura aquí planteada en tanto a nivel doméstico se promovía un ambicioso proyecto sociopolítico mientras que a nivel internacional se promovían crecientes ajustes fiscales que mermaban la soberanía de los estados. Para esto, creemos necesario entender el contrapunto que el Plan Austral significaba entre estas dos fuerzas sociales.

El trabajo se encuentra divido en dos partes, en la primera delimitamos las principales características económicas del contexto mundial y latinoamericano en los años ochenta. Específicamente, nos centramos en qué significaron aquellas transformaciones en los roles del Estado en relación a la sociedad y la definición de nuevas reglas para configurar los procesos de acumulación. En la segunda parte, abordamos el andamiaje político del proyecto democrático alfonsinista, la retórica constitutiva del mismo y su relación con el hostil contexto económico, especialmente durante 1985, entendido como un año de inflexión entre el reformismo alfonsinista y el neoliberal. 


\section{Macroeconomía mundial y transición democrática o reconfiguración capitalista sin bienestar social}

El gobierno de Alfonsín no fue un gobierno más entre los que sobrevinieron a los regímenes dictatoriales latinoamericanos, por el contrario, tuvo un carácter excepcional dado el profundo quiebre que la dictadura de 1976 produjo en la sociedad, especialmente en el régimen de acumulación (Basualdo, 2018). Además, los problemas internos que acarreó la vuelta de la democracia no pueden entenderse sin las más amplias transformaciones dadas en la economía mundial de los años ochenta (Boron y Gambina, 2002). De modo que un país que cargaba con una de las deudas latinoamericanas más grandes, combinadas con altas tasas de interés y caída de los precios de productos primarios a niveles internacionales, también sufría las secuelas de la apertura económica y la desregulación financiera que había dejado el que el plan económico del régimen anterior diseñado por el entonces ministro José Martínez de Hoz (1976-1981) (Brenta, 2019).

Así, la transición democrática se desarrolló en un complejo mecanismo de condicionamientos externos, como lo señalaron Alonso y Simonoff (2016):

Se contextualizó en un periodo de reestructuración aún inacabado del sistema económico internacional en sus múltiples dimensiones. En lo que refiere al ámbito de lo monetario-financiero (...) la gestión alfonsinista debió afrontar un escenario de crisis sistémica, en la que las normas e instituciones remanentes del denominado acuerdo de Bretton Woods (...) se mostraron incapaces de encauzar los desafíos concitados por la crisis de la deuda soberana de ciertos Estados periféricos (...) desatadas tras el incremento de la tasa de interés de Estados Unidos (p. 52) ${ }^{2}$.

Así, en los años ochenta el continente latinoamericano atravesó profundos cambios en la dinámica macroeconómica mundial que afectaron a los modelos de desarrollo nacionales producto, en verdad, de las transformaciones que atravesaba el mundo capitalista desde la década anterior con la crisis de los cánones del keynesianismo (Rougier y Odisio, 2018). Estos cambios pueden dividirse en dos: en primer lugar, los que involucraban a los principios macroeconómicos que daban sustento a las políticas económicas, políticas que pasaron a estar determinadas por la concepción neoliberal y que tenían el objetivo de estabilizar y reactivar las economías en circuitos comerciales abiertos al exterior (Ocampo, 2014; Stallings, 2014). Para ello, era nece-

2. Más generalmente las transformaciones mundiales del modelo de acumulación fordista surgido en la posguerra, aún sin la instalación de una dinámica clara de acumulación, provocaban políticas potencialmente restrictivas por parte de las potencias extranjeras, sobre todo en el ámbito comercial y de gran impacto en nuestro país, que solo serían revertidas con el auge del neoliberalismo en los años 1990. 
sario recomponer las tasas de ganancia percibidas por el capital privado a través de la reducción de los déficits fiscales y la emisión monetaria, la liberalización de precios y salarios, etc. Y, en segundo lugar, se encontraba la prioridad de impulsar la reforma neoliberal del Estado de bienestar, cambiando las viejas reglas que fueron hegemónicas en los estados de posguerra, apuntando a políticas sectoriales como las reformas estructurales $^{3}$. Entre las principales medidas asociadas al desarme del estado benefactor se encontraban la apertura y desregulación de los mercados; la privatización de los servicios públicos y de las empresas estatales y la implementación de tipos de cambios flexibles como estrategia ante las amenazas de retiros de capitales, reglas que en verdad habían reemplazado los antiguos acuerdo de Bretton Woods de posguerra durante los años setenta y que ahora comenzaban a erosionar al Estado debilitando sus capacidades políticas, burocráticas y administrativas (Cao, 2017; Nemiña, 2013)

Estas transformaciones se tornan fundamentales para entender el devenir del gobierno alfonsinista, dado que en la economía estos dos aspectos condicionaron el rol que el Estado adquirió en la política económica y el perfil de los planes diseñados para actuar sobre la situación socioeconómica. El cambio de paradigma concretado con el Consenso de Washington en los noventa ya se encontraba en franco avance durante los años ochenta, impulsando las reformas para mejorar la eficiencia en el uso de los recursos y el crecimiento económico. Así, la apertura comercial, la liberalización financiera y las privatizaciones, comenzaban a ganar lugar en la agenda de debates. También, reformas sectoriales en áreas como la salud y la educación estuvieron presentes y atravesadas por las tensiones entre el reformismo alfonsinista y el avance de apertura al mercado y participación privada ${ }^{4}$.

3. Barletta y Yoguel (2017) afirman que no existe consenso en torno a los conceptos que definen lo que se entiende por un cambio estructural, aún más, tampoco lo hay en lo que se refiere al concepto estructural. Sin embargo, nosotros aceptamos que el cambio estructural involucra específicamente a la modernización del aparato productivo con vistas a la promoción del crecimiento económico en los años analizados. Más allá del abanico de contribuciones teóricas por el lado de la heterodoxia en cuanto al tema, es necesario destacar los lineamientos más remarcados por esta: el cambio estructural a partir de la relocalización de la fuerza de trabajo hacia actividades de mayor productividad, como consecuencia del cambio tecnológico endógeno, como propiedad emergente del sistema económico y, finalmente, como resultado de procesos de coevolución entre la oferta y la demanda. No obstante, también entendemos que el avance del neoliberalismo comenzaba a ver en el cambio estructural otras necesidades que se relacionaban con la apertura comercial, financiera y las privatizaciones.

4. Por ejemplo, el congreso pedagógico nacional (1984-1988) y la creación del Seguro Nacional de Salud (1988) constituyeron iniciativas con una impronta alfonsinista que desembocaron en la década siguiente en reformas de acuerdo a los cánones neoliberales de los años del gobierno de Carlos Menem (1989-1990). Al respecto, pueden consultarse, para el primer caso (Wanschelbaum, 2014), y para el segundo (Repetto y Alonso, 2004). 
En aquellos años se desarrollaba una rediscusión acerca del antiguo rol del Estado en el terreno económico y social respecto de los viejos acuerdos que habían dominado la escena los últimos treinta años desde la posguerra. Así, el avance neoliberal preanunciado en algunos casos como el Chile con Augusto Pinochet (1973-1990) y en parte, con menor apoyo externo en Argentina durante desde 1975 y 1976, se relacionaba con la aceptación que esta doctrina comenzaba a ganar en los centros de poder mundial como con el neoconservadurismo en el Reino Unido y en Estados Unidos, países en los cuales los gobiernos liderados por Margaret Thatcher (1979-1990) y Ronald Reagan (1981-1989) establecieron un clima de ideas favorables a replegar las acciones estatales de toda la esfera económica y social que pudiera ser explotada por los agentes privados en el marco de la economía de mercado (Alonso y Simonoff, 2016; Rabotnikof, 2008).

En este marco, la reconstrucción de la democracia pasaba en gran medida por la reconstrucción de una sociedad civil plural y diversa. Las características del tipo de sociedad civil que se buscaba plasmar, habían surgido inicialmente en los países del Este europeo en el cuadro del derrumbe de los socialismos reales. Fue durante la década de 1980 en Europa y Estados Unidos que comenzó a sellarse la crisis del Estado de Bienestar y del liberalismo social frente al surgimiento de un nuevo modelo que marcaba el comienzo del neoliberalismo. Se presentó al mercado como organizador y regulador de las relaciones sociales, motorizado por gobiernos asociados a las derechas y focalizado en estándares internacionales construidos desde organismos que imponían la calidad, la eficiencia y el ajuste sobre las antiguas insignias del bienestar. Las críticas en torno a los gastos en servicios sociales que se habían realizado desde la posguerra apuntaban a la falta de calidad, la excesiva centralización y burocratización. Una de las soluciones que se proponían era devolver el control de áreas claves de la sociedad a los individuos y a la iniciativa privada como sinónimo de iniciativa ciudadana y ejercicio de la "libertad de elección" (Rabotnikof, 2008, p. 38). De modo que el alfonsinismo en este cuadro buscó aglutinar un sentimiento colectivo y de pertenencia para modernizar la sociedad de acuerdo a los valores democráticos que promovía como la ética de la solidaridad, la justicia distributiva y la democracia participativa (Boron, 2000), pero en un contexto en que el Estado como organizador de las relaciones sociales y económicas se encontraba cada vez más interpelado por los centros de poder mundial.

Las reformas, en palabras de Fair (2014) "promercado", buscaban transformar la raíz de la estructura económica y comercial del Estado de posguerra. Es decir, los Estados de Bienestar y la estrategia económica basada en la industrialización por sustitución de importaciones (ISI) eran cuestionadas desde los años setenta por políticas económicas de estabilización y ajuste estructural de orientación neoliberal sustentadas, principalmente, en la crítica a un Estado interventor, inflacionario, deficitario y 
burocrático, que debía ser reconfigurado en aras de la eficiencia, el ajuste fiscal y el disciplinamiento, ahora democrático, de la mano de obra.

La generación de reformas encaradas en los años ochenta se diferenciaron de otras iniciativas de reforma estatal y administrativa llevadas a cabo en Latinoamérica a lo largo del siglo XX (Naím, 1995). El clima ideológico que otorgaba al Estado un rol fundamental como articulador de las relaciones sociales iba a cambiar a partir de los ochenta, ya que comienza a verse cuestionado, proceso que generaba una serie de tensiones en el alfonsinismo entre un Estado que pretendía consolidar la democracia pero que, a su vez, debía redefinir su rol en las relaciones económicas y sociales según los cambios internacionales y el paradigma neoliberal predominante.

En este sentido, Oszlak (1999) sostiene que, "las reformas de primera generación tuvieron como objetivo encoger al Estado, no necesariamente mejorarlo" (p. 3). Es decir que el objetivo principal era desprenderse de segmentos institucionales, mientras que la racionalización de las estructuras estatales quedaría más bien para otra etapa que fue desarrollada posteriormente en la década de 1990. En los ámbitos laborales, reestructuración del gobierno, sistema judicial, económico-estatal, entre otras, fueron en los años de Alfonsín espacios de disputas a razón de los intentos por llevar a cabo reformas, aunque no siempre persiguiendo la estricta línea que aglutinaría el llamado Consenso de Washington (Chourio, p. 2003). De todas formas, las proyecciones reformistas y la constante desestabilización económica a la que esgrimían quienes eran partidarios de las mismas, chocaban con el presidencialismo de Alfonsín y el fortalecimiento estatal que se requería para consolidar la democracia ${ }^{5}$.

En este contexto, es posible comprender el neoliberalismo en los términos que lo definiera Forcinito (2010), como:

“... una corriente de pensamiento que surge en los años cincuenta como una reacción radical a la hegemonía keynesiana en el campo de la economía, y que combina elementos de la teoría neoclásica, y otras teorías afines, con una filosofía política que atribuye un valor fuertemente positivo a la 'desigualdad' como mecanismo de promoción de la competencia y, con ella, a la eficiencia en el sistema económico" (p. 6).

De modo que, siguiendo a la misma autora, el neoliberalismo no es homogéneo, sino que se compone de un conjunto de vertientes teóricas diferenciadas. Sin em-

\footnotetext{
5. En aquellos años, Rehren (2019) decía que los procesos de redemocratización latinoamericanos, especialmente el argentino, se encontraban desarrollando regímenes sólidamente presidencialistas que podría permitir una estabilización política compleja dirimiendo los conflictos entre los poderes militares que se oponían a la concertación democrática y las presiones corporativas políticas y económicas heredadas de los años previos. El autor afirma que los líderes de la transición buscaban encauzarse en un reformismo que les permitiera descentralizar el poder político con el objetivo de desarticular las posibilidades de una vuelta al autoritarismo.
} 
bargo, es posible aglutinar para los fines de nuestro trabajo que el neoliberalismo internacional de los años ochenta se tornaba pujante en cuatro aspectos. El primero de ellos era el que entendía a la desigualdad como un elemento que tornaba dinámicas a las sociedades, fundamento que inducía a desestimular los impulsos igualitaristas promovidos por los Estados de Bienestar. Segundo, la democracia sería un simple elemento de elección de gobernantes y no, por ejemplo, un sistema político capaz de sostener un modelo socioeconómico viable. Tercero, la intervención estatal era identificada como una productora de ineficiencias económicas y de las libertades personales frente a la verdadera libertad de mercado. Por lo tanto, respecto a este último punto, la intervención debía limitarse a determinadas áreas. Además, se reconocía que el mercado de competencia perfecta no existe, de forma que es en aquellas imperfecciones que el Estado debía intervenir para producir ese mercado ideal.

En nuestro país, como hemos mencionado, el régimen de dictadura de 1976 fue pionero en practicar las primeras reformas económicas asociadas al neoliberalismo con el plan llevado a cabo por el Ministro de Economía José Alfredo Martínez de Hoz, aunque las mismas estuviesen presentes desde muchos antes en nuestro país, como en América Latina (Morresi, 2008; 2010). En aquellos años, el contexto económico fue favorecido por la amplia disponibilidad de capitales, producto de la crisis del dólar primero y del petróleo posteriormente. Aunque las reformas hayan sido parciales y en casos abandonadas, produjeron amplios cambios en ámbitos como la política comercial y financiera, aunque en menor medida, por ejemplo, en la política tributaria. A este respecto, Forcinito (2004) sostuvo que:

“...la dictadura argentina promovió, en primer lugar, la profundización del proceso de concentración y centralización del capital sobre la base -fundamentalmente- de una brutal transferencia de ingresos desde el sector asalariado; en segundo lugar, el estancamiento y la desindustrialización de la economía, paralelamente a la financiarización e internacionalización de una porción significativa del capital controlado por la fracción dominante local y, en tercer lugar, el agravamiento de los desequilibrios macroeconómicos en el orden externo y fiscal derivados del creciente peso asumido por el endeudamiento externo, entre las principales transformaciones producidas" (p. 13) ${ }^{6}$.

6. También cabe aclarar que, en la misma línea, varios autores (Azpiazu, 1991; Azpiazu et al., 2004; Ortiz y Schorr, 2006) aclaran que durante el régimen militar esa desindustrialización implicó una "reprimarización" del sector manufacturero y una "simplificación productiva" en ramas industriales de un reducido procesamiento manufacturero y bajo dinamismo en el mercado mundial como la celulosa, el papel, los minerales no metálicos y los productos químicos. 
Luego, la crisis de la deuda y su correlato en el racionamiento del crédito voluntario para la región produjo un empeoramiento de las condiciones económicas a nivel continental. Las autoridades domésticas entendieron en un primer momento que se trataba de un choque transitorio, por lo que aumentaron las intervenciones estatales de diferentes formas, sin embargo, la crisis se prolongó afectando a las transiciones democráticas (Ocampo, 2014).

Con el tiempo y como respuesta al shock mexicano del año 1982, las economías latinoamericanas llevaron a cabo drásticos procesos de ajuste caracterizados por devaluaciones y medidas destinadas a reducir la absorción doméstica como el gasto público. Aunque los grados de éxitos en los procesos de ajuste latinoamericanos fueron variados, particularmente en la Argentina de 1983 el gobierno de Alfonsín asumió en un contexto difícil signado por el endeudamiento, el default, la inflación y una fuga de capitales que comprometían la estabilidad económica (Rapoport, 2012). Los problemas de diagnóstico y acción ante estos inconvenientes involucraban, en un comienzo, al programa político refundacional de Alfonsín apegado, en la estrategia económica, más a una visión del pasado desarrollista que a las transformaciones económicas contemporáneas (Pesce, 2006).

Como lo sostienen Damill y Fanelli (1994):

Los problemas básicos en los ochenta fueron el error diagnóstico respecto de la gravedad de los nuevos desequilibrios. Los países y los organismos que los asesoraban trataron de abordarlos, en un primer momento, con el herramental tradicional de estabilización. Esto es, con programas diseñados para tratar desequilibrios flujo/flujos transitorios entre el ingreso y la absorción, apoyados por montos de crédito externo insuficientes, apropiados quizás para situaciones transitorias de iliquidez, pero no para financiar prolongados procesos de cambio estructural" (p. 3).

Así, el primer programa económico de la democracia llevado a cabo por Bernardo Grinspun (1983-1985), luego de los intentos en conformar un club de deudores y revisiones de la deuda, acordó un stand by con el Fondo Monetario Internacional (FMI) que marcaría el principio del fin de las estabilizaciones tradicionales más relacionadas a la reactivación de la actividad económica mediante el incentivo de la demanda agregada. Alfonsín, y su proyecto refundacional democrática cedían así, luego del fracaso de un breve programa neokeynesiano, a las prescripciones de las instituciones financieras internacionales como a las presiones de los acreedores externos. Paradójicamente el programa de estabilización del FMI, centrado en límites a la emisión monetaria, al incentivo salarial y en aumentos de las tarifas de servicios públicos como las tasas de interés, no arrojó efectos positivos en economías con inflación estructural, altos endeudamientos, desfavorable evolución en los términos de intercambios y déficits públicos insostenibles, como consecuencia no solo de la deuda externa, sino de erogaciones estatales de larga data. 
De forma que, como parte de un proceso que se estaba configurando en nuestro país, comenzaron a perfilarse cuadros técnico-profesionales provenientes del campo económico (Heredia, 2006), que ganaban espacios en el poder político aun sin afiliaciones partidarias y que ofrecían diferentes diagnósticos para enfrentar la cuestión económica. Así, ante el fracaso en detener la inflación, el alfonsinismo buscaba respuestas en estos cuadros y adoptaba una nueva postura frente a los poderes internacionales, llevando a cabo "un giro realista" (Escudé y Cisneros, 2006, p. 168) en sus relaciones con Norteamérica como una nueva predisposición para rediseñar el capitalismo argentino (Wainer, 2010). A partir de este momento, que coincidiría con la formulación del llamado Plan Austral de 1985 como respuesta a la falta de solución de la cuestión económica por el lado de la heterodoxia primero como de la ortodoxia después, se configuraba en el alfonsinismo un nuevo poder político que apoyaba al presidente pero que no borraba las tensiones entre los radicales de tradición partidaria y los técnicos que se integraban (De Riz y Feldman, 1991). No obstante, la nueva redefinición política del alfonsinismo alteraría el programa refundacional frente a la nueva hegemonía del capital financiero motorizado en los organismos multilaterales de crédito decididos a reconfigurar nuevos modelos de acumulación en los países latinoamericanos (Morresi, 2008).

Luego del fracaso de Grinspun como del FMI, se hizo evidente que para cerrar la brecha fiscal y externa -como principales fuentes de desequilibrio de la economía- era necesario poner en efecto reformas estructurales que ampliaran el acceso al crédito y reordenaran los pagos externos, aunque necesariamente, antes debía frenarse la inflación. El Plan Baker desde 1985 fue una de las primeras iniciativas a nivel internacional, impulsadas por el FMI, el gobierno de los EE. UU y el Banco Mundial (BM), que ampliara el enfoque de la estabilización con reformas estructurales y que incluyera la cuestión, aunque retóricamente, de incentivar el crecimiento económico en la región. Así, con el Plan Austral de 1985, el ministro reemplazante de Grinspun, Juan Sourrouille, lanzaba una estrategia antiinflacionaria heterodoxa acordada con el FMI y que posteriormente desembocaría en las primeras iniciativas para implementar reformas que involucraron la privatización de empresas públicas y la apertura comercial. Fue en 1987 cuando junto a Rodolfo Terragno, entonces Ministro de Obras y Servicios Públicos, Sourrouille propusiera un ambicioso plan de redimensionamiento del Estado. Este intento de reformar el Estado fracasaría al no obtener el consenso necesario en el parlamento, pero constituyó un antecedente importante de la reforma del Estado desarrollada en su plenitud en la década menemista (Fair, 2008).

El anterior paradigma de desarrollo de los Estados de bienestar de posguerra comenzaba a ser paulatinamente así cuestionado frente al avance de un impulso globalizador que promovía patrones de apertura comercial y financiera a través de la política económica como prerrequisito para lograr una efectiva integración a las redes de 
comercio mundial y diseñar así una estrategia de crecimiento económico. Es decir, las crisis económicas pusieron de manifiesto el agotamiento de las condiciones que posibilitaban la estabilidad en las economías centrales de postguerra, abriendo paso a la necesidad de una reestructuración del capital por parte de las fracciones más poderosas (Forcinito, 2004).

De modo que los años 1980 fueron de profunda inestabilidad macroeconómica: alta inflación, corrientes déficits de cuenta corriente, endeudamiento externo público, desplazamiento del ahorro interno por el ahorro externo (fuga de capitales) y creciente debilidad del Estado. Este modelo, que había demostrado durante la dictadura una dinámica en ciclos cortos marcados por la disponibilidad de financiamiento externo condujo a que el Estado redujera su capacidad de intervención en el desarrollo productivo, pero también en la implementación de políticas de estabilización coherentes con ese desarrollo, lo que lo llevó paulatinamente a aceptar las recomendaciones ortodoxas, aunque con escaso éxito en implementar reformas económicas profundas.

\section{La transición a la democracia: un programa de refundación democrática}

En línea con el argumento de O’Donnell (1994), la construcción de un régimen democrático se caracteriza por dos fases. La primera es la que transita desde la descomposición del régimen anterior hasta la instalación de un nuevo orden (en este caso caracterizado en un primer momento por la celebración de elecciones libres y la transferencia de mando) y la segunda se relaciona con los mecanismos y dinámicas que convierten a un gobierno democrático en un régimen democrático. Es decir, el régimen modifica sus procedimientos de representación política, lo que conlleva que las conductas democráticas se tornen como los medios para la toma de decisiones y delimitación del poder.

En Argentina, la transición se caracterizó por la ausencia de un pacto formal entre el poder saliente y el entrante, derivado en última instancia del fracaso especialmente económico que produjo el plan económico de la dictadura, finalmente rebasado con la trunca guerra por las Islas Malvinas en 1982 y el efecto desfavorable a nivel internacional de la crisis de la deuda latinoamericana. Durante la fase final de la dictadura $y$ tras los fallidos intentos de negociaciones con el polo civil por imponer un proyecto que diera un conjunto de garantías al poder militar saliente, Alfonsín tuvo ciertas ventajas. Así lo expresó Masi (2014):

Una ventaja consistente es que Alfonsín se encuentra libre de compromisos y dotado de mayor autonomía, atento a la ausencia de un pacto en torno a la modalidad de abordar las cuestiones de Estado y la reconfiguración de la estructura socioeconómica del país (p. 26). 
La clave republicana liberal examinada en el discursivo alfonsinista durante la transición a la democracia puede ser entendida como lo definiera Ramírez (2019), es decir, como un discurso de tamiz regenerativo que, producido por el alfonsinismo, tenía la doble función de invitar a los actores a una resemantización de la política revisando las tradiciones previas y confiriendo a la UCR el liderazgo del proceso modernizador ${ }^{7}$. Así, desde los actos de campaña, Alfonsín recitaba el preámbulo de la Constitución Nacional y promovía la defensa del sistema republicano, representativo y federal, como de la igualdad de los ciudadanos ante la ley.

De modo que la democracia, característica primaria de la concepción de representación política, era para el alfonsinismo un método que conciliaba la modernización de las estructuras políticas y económicas con el progresivo aumento de la justicia social. De esta forma, sobre las bases de una democracia liberal y el cambio en las costumbres políticas se pretendía refundar una Segunda República (Sáez, 1994; Mercer, 2001), en alusión a la primera, iniciada con el ciclo político inaugurado desde la Constitución de 1853 que había conocido su auge en 1880 y durante las primeras décadas del siglo XX, pero había fracasado posteriormente en consolidar un régimen democrático estable a partir del ciclo de inestabilidad política en los años 1930. La UCR, en un afán hegemónico, ocupaba un lugar privilegiado al considerarse mediante el lenguaje democrático que promovía la recuperación de aquellas tradiciones olvidadas y despreciadas por las encarnizadas luchas por el poder emprendidas por las corporaciones en el pasado. Así, y con una propia genealogía partidaria se presentó como la alternativa y la protagonista de la transición democrática. Con democracia participativa, decía el presidente, se entendía a un movimiento para expandir los espacios de libertad recuperando la solidaridad y el sentido nacional (Alfonsín, p. 1987) y con la ética de la solidaridad, que conciliaba el principio de la libertad del liberalismo y el de la igualdad del socialismo, aseguraba que "el valor de la libertad depende de cómo ella está distribuida y el valor de la igualdad depende de qué es lo que se distribuye en forma igualitaria (p. 135).

No obstante, deben considerarse otras interpretaciones de este proceso como las de Campione y Muñoz (1994), quienes cuestionaran el aferramiento a una concepción de la democracia entendida en sus reglas procedimentales desvinculada de la organización social. Los autores, en esta línea, recomiendan considerar el desarrollo del régimen político en relación con las configuraciones sociales, económicas y culturales, dado que los análisis donde predomina la visión procedimental desvinculan el grado de legitimidad de un gobierno con la satisfacción de necesidades ciudadanas como la distribución de la riqueza, la posibilidad de movilidad social ascendente y la

7. Estos cambios en la cultura política se producían en un marco general de transformación de la cultura política en donde ahora, los partidos mayoritarios retomaban la importancia antes desbordada por otras formas de participación, en un proceso de partidización de la política (Birle, 1994). 
calidad de vida. Así, 1983 funcionaría como la insignia de la recuperación democrática tras largos años de desprecio frente a las expectativas de revertir la herencia del régimen anterior, donde el alfonsinismo a pesar de haber interpelado el anhelo de las masas, también favoreció la recomposición de un bloque dominante que se había estructurado durante los años previos.

También en este sentido, en referencia al papel de las fuerzas armadas, que interpretó Canelo (2013), estas se constituían como un factor central que tras abandonar el poder arbitral que habían tenido anteriormente se replegaban en sus demandas corporativas en el nuevo contexto democrático, generando importantes tensiones en todo el periodo. Estos límites, son articuladamente analizados por Pucciarelli (2006), quien entendió que la concatenación de sucesos y tensiones entre el poder democrático y el corporativo militar desembocó en el levantamiento de Semana Santa en 1987. Así, según la interpretación del autor, la posterior Ley de Obediencia Debida, que otorgaba la amnistía a los oficiales con grado menores que el de coronel, fue la culminación de un proceso de tensiones donde el alfonsinismo optó por no confrontar con el poder militar a pesar del amplio apoyo civil que había recibido en defensa de la democracia.

En este cuadro de tensiones, la idea de democracia participativa y solidaria promocionada por el alfonsinismo era opuesta a una democracia elitista en tanto proponía incluir a todos los actores en los procesos decisorios, aunque resulta claro que desde un comienzo la plural participación va a ser confusa frente a la denuncia de las corporaciones, especialmente los sindicatos peronistas, con las que a lo largo del gobierno se tuvieron ásperas relaciones como consecuencias del intento de reforma de normalización sindical, destinado en gran medida a desarticular el poder gremial de la oposición justicialista, promovida por el ministro de Trabajo Antonio Mucci en $1984^{8}$.

8. Merece una mención el fracaso de la Ley de Reordenamiento Sindical de Mucci, que tenía como objetivo democratizar los sindicatos proponiendo el voto directo, la representación de las minorías y las elecciones inmediatas y sin proscripciones en todos los niveles bajo control oficial. La ley se encontró con la oposición de los senadores peronistas (también el peronismo era mayoría en los gobiernos provinciales, lo que producía aún más descontento opositor con el sector del trabajo e inclusive propiciando que éste se acercara al sector empresarial) (Aruguete, 2006). A partir del fracaso de dicha ley se visualizaría un cambio en la estrategia política confrontativa que suponía, hasta aquel entonces, la lucha corporativista desarrollando instancias que dentro de los límites impuestos por la UCR buscarían lograr acuerdos sectoriales. Durante la gestión Grinspun, específicamente en junio y agosto, se firmaría el Acta de Coincidencias y luego se generaría una instancia llamada la Concertación Económica y Social con representantes del gobierno, de los sectores laborales y empresarios. Desde luego, se reclamaba que el Estado satisfaga las demandas sectoriales sin generar daños colaterales y finalmente la CGT terminó abandonado la mesa de concertación endureciendo su oposición al gobierno de Alfonsín. Posteriormente se produjo una singular alianza entre empresarios y la CGT, quienes firmarían el Documento de los 20 puntos donde se exigía un redimensionamiento del Estado empresario y cambios en las políticas cambiarias y arancelarias (Pesce, 2006). 
De esta forma, partimos de la convicción de que el gobierno de Alfonsín atravesó una tensión constante producida por la grave crisis económica y la necesidad coyuntural de institucionalizar un nuevo régimen. La figura de Alfonsín, envuelta en un carácter carismático con bases de apoyo en la ciudadanía que siguió creciendo, al menos hasta 1986, funcionó como el componente legitimador del contexto político. Como Sáez (1989) dijera en aquel entonces, la administración de la crisis bajo el personalismo alfonsinista -pero con un escaso consenso entre actores políticos y económicos- fue un problema para consolidar la llamada por el gobierno Segunda República en tanto plan integral de redefinición institucional en varias áreas de la sociedad. Principalmente porque el concepto de democracia participativa defendida por el alfonsinismo se limitaba a instalar un conjunto de reglas básicas y procedimentales para institucionalizar la democracia (Ramírez, 2019). Así, la democracia como proyecto político entre una dimensión procedimental y el personalismo de Alfonsín, entraban en tensión en tanto en el primer aspecto se requerían alianzas coyunturales con actores políticos que se oponían al gobierno, especialmente a su estrategia económica, como los sindicatos y algunos sectores económicos (Fair, 2013). En el segundo aspecto, el alfonsinismo se aislaba paulatinamente de los actores políticos y económicos, pues el justicialismo ejercía una férrea oposición al igual que los sindicatos como una gran parte del poder económico. Sin embargo, este buscaría avanzar en la búsqueda hegemónica para consolidar un tercer movimiento histórico (Díaz, 1984).

Cuando Alfonsín llegó al poder, la lectura era que el pasado político de medio siglo se presentaba como un obstáculo que la transición a la democracia debía superar. La eliminación del autoritarismo, la estabilización del régimen político, la supresión de las proscripciones de los partidos, el respeto por los derechos humanos y la modernización de la sociedad, especialmente del Estado, fueron parte del ethos del gobierno. Ese gesto refundacional se encargaba de definir una frontera política entre un pasado reciente repudiado y la construcción de un futuro que, en última instancia, explican el énfasis reformista (Dagatti, 2016; Vitale y Dagatti, 2016)9.

Quizás al respecto convenga recuperar la hipótesis de Barros (2002) quien ha señalado que uno de los fracasos del alfonsinismo en el terreno sindical fue no haber logrado ni disciplinar a los sindicatos, por un lado, ni incluirlos posteriormente en el espacio definido por la democracia alfonsinista, por el otro. Esto, en última instancia fue dándole espacios al peronismo para disputar la hegemonía política y con el deterioro económico que recurrentemente no perdía oportunidad de denunciar y donde encontró una plataforma favorable para la competencia electoral.

9. De hecho, el espíritu refundacional de aquellos años también alcanzaba al espacio público con la circulación del documental histórico de raíz radical La República Perdida de Miguel Pérez que fue convertido en una herramienta elemental para interpretar la historia reciente argentina en aquellos años. En la visión que transmitía este film la democracia era entendida como un valor absoluto respetuoso de los intereses nacionales y populares y se construía así un optimismo respecto del futuro institucional de país. Partiendo desde el yrigoyenismo de principios del siglo XX el film exalta la participación del pueblo y la odiosa oligarquía que desde 1930 con golpes militares habría 
Estas características del espíritu refundacional encarnado por Alfonsín van a entrar, luego del fracaso neokeynesiano de Grinspun, en una especie de ambivalencia. A partir de allí se convocó a un equipo de técnicos para formular un plan antiinflacionario. El Plan Austral anunciado en junio de 1985 constituyó un momento de ambigüedad con el proyecto político al abrir la posibilidad de implementar reformas en la economía de corte ortodoxa aunque paradójicamente se trataba de un plan heterodoxo en algunos aspectos como en la concepción de la inflación y la posibilidad de consolidar el crecimiento económico y mantener los pagos al exterior ${ }^{10}$. Este cambio manifestaba el progresivo acercamiento a ciertas posturas ortodoxas en la dimensión de la política económica, como a la necesidad de ajuste fiscal y pago de la deuda externa, frente al empeoramiento de las condiciones económicas tensionando la relación entre el bienestar social propiciado por la transición alfonsinista y la reconfiguración capitalista mundial que sucedía. No obstante, los planes económicos en esta etapa no pueden dejar de ser entendidos sin esta lógica que atravesó a la sociedad y al reformismo impulsado por el Estado en varias áreas y que quizás puedan ser encuadrado en un marco de redefinición de las fuerzas políticas nacionales que comenzaban a replantearse cambios en sus supuestos económicos, producto de las sucesivas crisis y la presión internacional.

En medio de esta tensión económica el alfonsinismo buscaba sentar las bases de organización democrática, materializadas en un intento de reforma constitucional, de traslado de la capital federal como de una reforma administrativa (Sáez, 1994), entre otras; que sirvieran tanto para consolidar la hegemonía alfonsinista como para gestionar las tensiones sociales propias de un sistema político con contradicciones. Finalmente, ante el aislamiento y el progresivo deterioro económico, estas iniciativas se perderían en su abandono o la falta de consenso para implementarlas, refugiándose así en una parcial institucionalización del régimen democrático (Campione, 2003). Fundamentalmente, este credo con características de un gran acuerdo, era pensado por el presidente para resolver la crisis del sistema político, lo que explica el llamado a la participación y ejercicio de la ciudadanía en la vida pública como parte de discurso alfonsinista (Alfonsín, 1980). Aunque los contenidos del gran acuerdo que se planteaba no estaban muy claramente definidos, se entendía que debía existir un consenso en torno a las normas democráticas para la superación política de una situación que

\footnotetext{
condicionado el desarrollo de la república hasta los años setenta. Finalmente, en 1986 cuando se estrenara La República Perdida II, también bajo la dirección de Miguel Pérez, se analizaría el retorno del peronismo en 1973, poniendo especial foco en los años de autoritarismo para finalmente arrojar la reflexión final de conciliación en aras de la vida nacional. Estos films manifiestan en cierta medida la reinstalación del debate en una sociedad que pretendía revisar el doloroso pasado reciente enfatizando en la importancia de los significantes de república y democracia (Montes, 2018).
} 10. Para más detalles sobre el Plan pueden consultarse Frenkel y Fanelli (1987) y Machinea (1988). 
se entendía como dislocada, aunque finalmente esta no alcanzara a equiparar la presión internacional y la falta de interés de grupos locales en formular una estrategia de desarrollo que permitiera acabar con la inflación en una crítica situación externa (Barros, 2002).

Sin embargo, desde un comienzo, la voluntad reformista del gobierno se sustentó en los objetivos de consolidar la democracia y modernizar el Estado. Estos dos aspectos, resultaron centrales para comprender la relación entre la orientación política de la gestión del gobierno y los planes económicos que dialogaron con esa realidad. Reformar las instituciones del Estado como parte de la modernización, fue un aliciente central para consolidar la democracia y que, a su vez, involucró la intervención del Estado en las políticas económicas mediante planes específicos, pero desde 1985 otro reformismo avanzaba en tono a las recomendaciones internacionales. Es por ello que la transformación y la reforma estuvieron presentes en el proyecto político, y desde un comienzo involucraron la redefinición del papel del Estado, la racionalización de los recursos y la descentralización como medidas para garantizar una verdadera viabilidad, pero desde la política económica se contraponía con el reformismo ortodoxo y neoliberal que alcanza un auge internacional. Desde el Norte se iniciaba un periodo caracterizado por poner fin al control de movimientos de capitales con el extranjero, es decir, liberalizando los sistemas financieros internacionales como forma de poner en marcha un movimiento de desregulación monetaria y financiera que condujera a una rápida expansión de los mercados de obligaciones interconectados entre las naciones permitiendo la aparición de un sistema financiero mundial acorde a la finaciarización (Basualdo, 2006; Chesnais, 2001).

Es por ello que, en este contexto y al margen de la necesidad de estabilización económica que motivó la puesta en marcha del Plan Austral, el mismo no puede disociarse del programa reformista más amplio del alfonsinismo. El Plan Austral, además de ser un plan de estabilización centrado en su eje antiinflacionario, fue un programa de reforma económica con una matriz caracterizada por el proyecto democrático refundacional, dado que se aferraba a la defensa de la democracia, pero que a su vez disputó márgenes de acción a la ortodoxia. No obstante, con el transcurso de su deterioro cedió a las iniciativas ortodoxas alejándose de su formato original en una constante disputa entre los actores internacionales de poder como el Banco Mundial, el FMI y los grupos económicos locales fortalecidos tras la dictadura (Azpiazu et al., 2004).

El Plan Austral formó lo más alto de esta ambivalencia, cuando luego de fracasar el Plan Grinspun se hiciera evidente que la refundación democrática debía avanzar también en el plano de la economía si se quería estabilizar la democracia. El Plan Austral constituye un momento de ambivalencia en tanto, generaba tensiones entre la aceptación de la necesidad de estabilización económica con normas ortodoxas y la propuesta contemporánea de los promotores del gobierno radical Nun (1987) y Por- 
tantiero $(1987)^{11}$. Éstos, en una disputa por caracterizar el desarrollo político alfonsinista, reflexionaban sobre la posibilidad de un socialismo no opuesto al capitalismo en el que pudiera avanzar la democracia y el Estado de derecho como canalizadores de las manifestaciones populares en el devenir de las transiciones democráticas (Ramírez, 2019). Entonces, especialmente la propuesta de intervención estatal en la economía desde el Plan Austral refleja un momento reformista donde se buscó reorganizar diversas áreas como empresas, sistema de salud, sistema educativo, sistema judicial, reforma territorial, etc. Así, las transformaciones sociopolíticas que caracterizaron a la transición a la democracia y las motivaciones de la UCR en ese contexto se entremezclaron abriendo una disputa por qué tipo de reformismo ganaría espacios (Carlés, 2001).

Finalmente, el Plan Austral presentó un paulatino fracaso al volver la inflación y no definir reformas estrictamente económicas dadas las presiones que se enfrentaban. En este sentido, influyeron los endebles acuerdos con los grupos económicos locales y la constante presión por los ajustes ortodoxos que manifestaron el avance del neoliberalismo en la región (Balsa, 2001). Luego, el Plan Primavera en 1988 ya enmarcado en la cesación de pagos, intentó un nuevo congelamiento de precios con un desdoblamiento del mercado cambiario que generó un cambio de las alianzas política-económicas y una relación aún más tensa con el impulso neoliberal que lograba instalarse en el discurso de los funcionarios del gobierno en tanto ahora se reivindicaban abiertamente las reformas estructurales de corte neoliberal y ajuste fiscal. Así, la promesa de la democracia liberal, reformulada incluso desde sectores reformistas de izquierda que se vincularan con Alfonsín desde su mandato, fue perdiendo impulso también en el área de la política económica (Basombrío, 2014). En este marco, la relación entre estabilización y reformas estructurales constituye un punto central para comprender el devenir del Plan Austral y la política económica en particular. Estabilizar la economía en un contexto de inercia inflacionaria y deterioro de las variables

11. Como lo explica Freibrun (2014) las contribuciones de Portantiero, Nun, Emilio de Ípola, Atilio Boron, Guillermo O’Donnel, Margarita Graziano, Pablo Guissani, Sergio Bufano y Carlos Strasser, daban cuenta desde la izquierda cultural de la construcción de un tiempo histórico donde la democracia tomaba un valor en sí mismo tomando como punto de partida la política. El asesoramiento de círculos intelectuales de que gozó el alfonsinismo daba cuenta de un proceso que desde la segunda mitad del siglo XX venía politizando a los intelectuales y propiciando su participación pública. En los años 1980 éstos se volcaron a la búsqueda de guías políticas para la institucionalidad democrática. Basombrío (2014) ha señalado que la base de estos apoyos estuvo, por un lado, en el Grupo de Carlos Nino que fomentaba un liberalismo igualitario y, por el otro, en el Grupo Esmeralda fundado por el empresario Goodbar Meyer y Eduardo Issaharoff, que defendía una democracia desde la redefinición de la izquierda, es decir, a favor del Estado de Derecho y las libertades. Ambos grupos coincidían en que era primordial fundar una nueva política para la transición y entendían que estaban en una etapa fundacional y alimentaban la lectura culturalista que entendía que la estabilidad democrática asociada al bienestar social. 
macroeconómicas domésticas y un hostil contexto internacional se tornó en un condicionamiento central para un gobierno que pretendió formular un programa de refundación democrática que finalmente quedó empantanado entre la falta de acuerdos sectoriales como el desvanecimiento paulatino de la hegemonía alfonsinista abriendo espacios ante la ortodoxia neoliberal y los mecanismos de mercado.

\section{Reflexiones Finales}

El proyecto alfonsinista buscó sentar nuevas bases para un régimen democrático que, lejos de ser objetivo, tenía como protagonista a la UCR y el personalismo alfonsinista. En verdad, esta propuesta se contradecía en algún punto con la anunciada reactivación de una pluralidad democrática que encontraba límites ante la lucha contra el indeseado corporativismo del pasado reciente desestabilizaba las reglas elementales de la democracia argentina. No obstante, durante la década de los años 1980 el paulatino avance del neoliberalismo en el marco de una reconfiguración capitalista mundial reorientada a la financiarización económica imponía amplios condicionantes a partir de la creciente injerencia de los organismos financieros internacionales. Además, un contexto externo sumamente perjudicial para la Argentina como lo eran el deterioro de los términos de intercambio y las altas tasas de interés inducidas por la política económica estadounidense funcionaba como una plataforma de impedimentos para la transición.

Asentar la democracia institucional, pero propiciando el bienestar social, fueron parte de las ideas centrales de la inmediata transición democrática y el proyecto refundacional alfonsinista. Sin embargo, ante los problemas sociopolíticos desde el fracaso de su primer plan económico como del FMI en estabilizar las variables básicas, se construiría una relación más dinámica con los poderes internacionales. En este sentido, el consenso que instalaba el Plan Austral puede ser entendido como la inevitable convergencia de un contexto macroeconómico mundial de transformaciones que el capitalismo global atravesada desde los años 1970 y que ahora, con la crisis de la deuda latinoamericana, veía un terreno propicio para echar las bases del neoliberalismo internacional en la región. En este sentido fue que el Plan Austral el que puso en cuestión el proyecto modernizador de Alfonsín en tanto lo contrariaba con las necesidades de ajustes fiscales que se imponían a través de las recomendaciones internacionales. En suma, entendemos el año 1985 funcionó como un momento de ambivalencia dado que el Austral tenía la doble faceta de defender la democracia y otorgar cierto consenso al ajuste ortodoxo. Así, y al no avanzar el Austral en reformas económicas de fondo tras reducir significativamente los índices de inflación, paralizó el proyecto refundador del alfonsinismo. Por consiguiente, este último entraba en tensión con la vuelta de la inflación, los persistentes déficits fiscales y las complejas renegociaciones de la deuda que en 1988 acabarían en una moratoria de hecho 
jaqueando el plan refundador inicial y propiciando el abandono de las promesas de bienestar social.

\section{Referencias}

Aboy Carlés, G. (2001). Las dos fronteras de la democracia argentina: la reformulación de las identidades políticas de Alfonsin a Menem. Rosario: Homo Sapiens.

Alfonsín, R. (1980). La cuestión argentina. Buenos Aires: Torres Agüero Editor.

Alfonsín, R. (1987). «Texto del documento entregado por el presidente Alfonsín». Neudelman, R. (comp.) Raúl Alfonsín. El poder de la democracia (pp. 127-170). Buenos Aires: Fundación Plural.

Alonso, J., y Simonoff, A. (2016) «El gobierno de Raúl Alfonsín (1983-1989): aproximaciones respecto al interjuego de la estrategia de inserción internacional, modelo de desarrollo y política exterior». En: Busso, A. (cord.) Modelos de desarrollo e inserción internacional. Aportes para el análisis de la politica exterior argentina desde la redemocratización (1983-2011) (pp. 49-123). Rosario: UNR.

Aruguete, E. (2006). «Lucha política y conflicto de clases en la postdictadura. Límites a la constitución de alianzas policlasistas durante la administración de Alfonsín». En: Pucciarelli, A. (comp.) Los años de Alfonsín ¿El poder de la democracia o la democracia del poder? (pp. 413-460). Buenos Aires: Siglo XXI.

Azpiazu, D. (1991). «Programas de ajuste en la Argentina de los años ochenta: ¿década perdida o decenio regresivo?». Ponencia presentada en el Seminario "Ajuste económico, sindicalismo y transición política en los años ochenta, organizado por el Memorial de América Latina, San Pablo. Recuperado de https://n9.cl/gpkek.

Azpiazu, D., Basualdo, E., y Khavisse, M. (2004). El nuevo poder económico en la Argentina. Buenos Aires: Siglo XXI.

Balsa, J. (2001) «El Estado democrático y la gobernabilidad. Sus efectos en la sociedad y en la economía». En: Gibal de Blacha, N. (Cord.) Estado, sociedad y economía en la Argentina (pp. 1930-1997). Buenos Aires: UNQUI.

Barletta, F. y Yoguel, G. (2017) «De qué hablamos cuando hablamos de cambio estructural?» En: Abeles, M.; Cimoli, M. y Lavarello, P. (eds.) Manufactura y cambio estructural. Aportes para pensar la politica industrial en la Argentina (pp. 27-54). Santiago: CEPAL.

Barros, S. (2002). Orden, democracia y estabilidad. Discurso y política en la Argentina entre 1976 y 1991. Córdoba: Alción.

Basombrío, M. (2014) «Intelectuales y poder: la confluencia del socialismo-liberalismo durante la presidencia de Alfonsín». Passagens. Revista Internacional de Historia Política e Cultura Jurídica, 6(2): 376-398. Recuperado de https://n9.cl/ vnrmn. 
Basualdo, E. (2006). La reestructuración de la economía argentina durante las últimas décadas de la sustitución de importaciones a la valorización financiera. Buenos Aires: CLACSO.

Basualdo, E. (2018). Endeudar y fugar. Un análisis de la política económica de Martínez de Hoz a Macri. Buenos Aires: Siglo XXI.

Birle, P. (1994). Los empresarios y la democracia en la Argentina. Conflictos y coincidencias. Buenos Aires: Editorial de Belgrano.

Boron, A. (2000) «Los dilemas de la modernización y los sujetos de la democracia». En: Boron, A. (comp.) Tras el búho de Minerva. Mercado contra democracia en el capitalismo de fin de siglo (pp. 89-97). Buenos Aires: CLACSO.

Boron, A., y Gambina, J. (2002). La globalización económico financiera. Su impacto en América Latina. Buenos Aires.

Brenta, N. (2019). Historia de la deuda externa argentina. De Martínez de Hoz a Macri. Buenos Aires: Capital Intelectual.

Campione, D., y Muñoz, J. (1994). El Estado y la sociedad de Alfonsín a Menem. Buenos Aires: Letra Buena.

Campione, D. (2003). «Una democracia que se consolida degradándose. Aportes para el Estado y la Administración Gubernamental. Participación, democracia y gobernabilidad». Recuperado de https://n9.cl/z8h5.

Canelo, P. (2013). «¿Qué hacer con las fuerzas armadas? Treinta años de cuestión militar en la Argentina». Observatorio Latinoamericano, 12, 136-148.

Cao, H. (2017).«Reforma estatal y provincias en el sexenio alfonsinista». Revista Perspectivas de Políticas Públicas, 6(12): 275-303. Doi: https://doi.org/10.18294/ rppp.2017.1510.

Chesnais, F. (2001). La mundialización financiera. Génesis, costo y desafíos. Buenos Aires: Lozada.

Chourio, J. (2003) «De la primera a la segunda generación de reformas del Estado en América Latina; giro ideológico y cambio conceptual». Cuadernos de Economía, 22(38), 95-125. Recuperado de https://n9.cl/jvhin.

Damill, M., y Frenkel, J. M. (1994). La macroeconomía de América Latina: de la crisis de la deuda a las reformas estructurales. Buenos Aires: CEDES.

Dagatti, M. (2016). «La refundación de la democracia argentina. La retórica constitutiva del discurso inaugural del presidente Raúl Alfonsín». African Yearbook of Rhetoric, 7(3), 11-19. Recuperado de https://n9.cl/f7j94.

De Riz, L., y Feldman, J. (1991). El partido en el gobierno: la experiencia del radicalismo 1983-1989. Documento 64. Buenos Aires: CEDES. 
Díaz, J. A. (1984). Las alternativas radicales. El proyecto movimientista sobrevuela la UCR. El Periodista de Buenos Aires, 1 (11), 2-6.

Escudé, C., y Cisneros, A. (2000). Historia de las relaciones exteriores argentinas. Buenos Aires: CARI.

Fair, H. (2008). «El proceso de reformas estructurales en Argentina. Un análisis del primer gobierno de Menem». Revista OIKOS, 12(25), 35-49. Recuperado de https://n9.cl/ocfz1.

Fair, H. (2013). «El desvanecimiento del discurso nacional-popular en la Argentina (1988-1993)». Entramado, 9(2), 118-137. Recuperado de https://n9.cl/1104.

Fair, H. (2014). «Las reformas pro-mercado en Argentina. Un análisis sociohistórico de las políticas económicas del periodo 1976-1989». Revista Electrónica del Programa de Maestrado em Desenvolvimiento Regional da Universidade do Contestado, 4(1), 132-164.

Forcinito, K. (2004). «La intervención del Estado argentino en los sectores privatizados de infraestructura e hidrocarburos durante los años noventa: impactos sobre la relación entre el capital y la fuerza de trabajo» (Tesis de maestría). Buenos Aires: FLACSO.

Forcinito, K. (2010). «Aproximación al estudio del pensamiento económico predominante en la Argentina desde los años setenta: la recepción doméstica del pensamiento neoliberal». Asociación argentina de Historia Económica, Universidad Nacional de Río Cuarto. XXII Jornadas de Historia Económica, Córdoba, 21-24 de septiembre.

Freibrun, N. (2014). La reinvención de la democracia, intelectuales e ideas políticas en la Argentina de los 80. Buenos Aires: Imago Mundi.

Frenkel, R., y Fanelli, J. (1987). «El Plan Austral: un año y medio después». El Trimestre Económico, 54, 55-117.

Heredia, M. (2006). «La demarcación de la frontera entre economía y política en democracia. Actores y controversias en torno de la política económica de Alfonsín». Pucciarelli, A. (comp.) Los años de Alfonsín: ¿el poder de la democracia o la democracia del poder? (pp. 101-150). Buenos Aires: Siglo XXI.

Machinea, J. (1988). Stabilization under Alfonsin's government: a frustrated attempt. Buenos Aires: CEDES. Document N. 62.

Masi, A. (2014). Los tiempos de Alfonsín. Buenos Aires: Capital Intelectual.

Mercer, M. (2011). «Transición y consolidación democrática en la Argentina: una lectura desde la intelectualidad» (Tesis de Licenciatura). La Plata: Universidad Nacional de la Plata. Recuperado de https://n9.cl/hnf59. 
Montes, V. (2018). «El uso de imágenes de archivo para representar la Historia y las multitudes populares en el cine de la transición democrática». Revista de la Asociación Argentina de Estudios de Cine y Audiovisual, 18, 228-250. Recuperado de https://n9.cl/tswri.

Morresi, S. (2008). La nueva derecha argentina. La democracia sin política. Los Polvorines: UNGS.

Morresi, S. (2010). «Apuntes preliminares para un estudio del neoliberalismo en Argentina». En E. Muraca, R. Romanin y T. Groth(comp.) Teoría y práctica de la politica. Argentina y Brasil (pp. 299-319). Los Polvorines: UNGS.

Naím, M. (1995). «Latinoamérica en la segunda fase de la reforma». Revista Occidental, 12(2), 213-234.

Nemiña, P. (2013). «El FMI y la política económica argentina». En: Dossier Argentina: 30 años de democracia (pp. 150-163). Buenos Aires: Observatorio Latinoamericano 12 .

Nun, J. (1987). «La teoría política y la transición democrática». En: Nun, J. y Portantiero, J. (comp) Ensayos sobre la transición democrática Argentina. Buenos Aires: Puntosur.

Ocampo, J. (2014). «La crisis latinoamericana de la deuda a la luz de la historia». En J. Ocampo, B. Stallings, I. Bustillo, H. Velloso y R. Frenkel (comps.) La crisis latinoamericana de la deuda desde la perspectiva histórica (pp. 19-49). Santiago de Chile: CEPAL.

O’Donnell, G. (1994). Democracia delegativa. Journal of Democracy en español, 5(1), 55-69. Recuperado de https://n9.cl/1t53x.

Ortiz, R., y Schorr, M. (2006). «La economía política del gobierno de Alfonsín: creciente subordinación al poder económico durante la década perdida». En A. Pucciarelli (Coord.). Los años de Alfonsín. ¿el poder de la democracia o la democracia del poder? (pp. 291-333). Buenos Aires: Siglo XXI.

Oszlak, O. (1999). «De menor a mayor: el desafío de la "segunda” reforma del Estado». Revista Nueva Sociedad, 160, 1-17. Recuperado de https://n9.cl/6i6h.

Palermo, V. (2004). «Entre la memoria y el olvido: represión, guerra y democracia en Argentina». Revista de Investigaciones Ibéricas y Latinoamericanas, 10, 2, 131 148. Doi: 10.1080/13260219.2004.10426799.

Pesce, J. (2004). «La gestión del ministro Grinspun en un contexto de transición democrática: errores de diagnóstico y subestimación del poder económico local e internacional». Ciclos, 14(28), 65-88. Recuperado de https://n9.cl/4y2m. 
Pesce, J. (2006). «Política y economía durante el primer año del gobierno de Alfonsín. La gestión del ministro Grinspun». En A. Pucciarelli (Coord.) Los años de Alfonsín ¿el poder de la democracia o la democracia del poder? (pp. 236-271). Buenos Aires: Siglo XXI.

Portantiero, J. (1987). «La concertación que no fue: de la Ley Mucci al Plan Austral». En J. Portantiero y J. Nun (comps) Ensayos sobre la transición a la democracia en la Argentina (pp. 139-173). Buenos Aires: Punto Sur.

Pucciarelli, A. (2006). «La República no tiene ejército: el poder gubernamental y la movilización popular durante el levantamiento militar de Semana Santa». En: A. Pucciarelli (coord.) Los años de Alfonsín ¿el poder de la democracia o la democracia del poder? (pp. 114-149). Buenos Aires: Siglo XXI.

Rabotnikof, N. (2008). «Lo público hoy: lugares, lógicas y expectativas». Iconos. Revista de Ciencias Sociales, 32, 37-48. Recuperado de https://n9.cl/z7hyt.

Ramírez, V. A. (2019). La democracia como mandato. Radicalismo y peronismo en la transición argentina (1980-1987). Buenos Aires: Imago Mundi.

Rapoport, M. (2012). Historia económica, politica y social de la Argentina, 1880-2003. Buenos Aires: Emecé.

Rougier, M., y Odisio, J. (2018). “Argentina será industrial o no cumplirá sus destinos”: las ideas sobre el desarrollo nacional (1914-1980). Buenos Aires: Imago Mundi.

Rehren, A. (2019). «Liderazgo presidencial y democratización en el Cono Sur de América Latina». Revista de Ciencia Política, 14(1-2), 63-87. Recuperado de https:// n9.cl/htncd.

Repetto, S., y Alonso, G. V. (2004). «La economía política de la política social argentina: una mirada desde la desregulación y descentralización». Serie políticas sociales 97. Santiago de Chile: CEPAL. Recuperado de https://n9.cl/u1kmc.

Sáez, A. (1989). «Democracia, alternancia y crisis en Argentina». Revista de Estudios Políticos, 66, 263-297. Recuperado de https://n9.cl/94aa7.

Sáez, A. (1994). «¿Por qué no la Segunda República argentina?» América Latina Hoy. Evsalrevistas, 7, 81-87. Recuperado de https://n9.cl/gb4si8.

Stallings, B. (2014). «La economía política de las negociaciones de la deuda: América Latina en la década de los ochenta». En J. Ocampo, B. Stallings, I. Bustillo, H. Velloso y R. Frenkel (comps.) La crisis latinoamericana de la deuda desde la perspectiva histórica (pp. 53-81). Santiago de Chile: CEPAL.

Vitale, M., y Dagatti, M. (2016). «La construcción de la Argentina democrática en el discurso de asunción del presidente Alfonsín». HALLAZGOS, 13(26), 65-91. Recuperado de https://n9.cl/sgvzr. 
Wainer, A. (2010). «La primera etapa del régimen de valorización financiera. Parte II (1983-1989)». En: AA. VV. Desarrollo económico, clase trabajadora y luchas sociales en la argentina contemporánea (pp. 89-108). Buenos Aires: IEC-CONADU. Wanschelbaum, C. (2014). «La educación durante el gobierno de Raúl Alfonsín (Argentina, 1983-1989)». Ciencia, Docencia y Teconología, 25(48), 75-112. Recuperado de https://n9.cl/ts7om.

Zoppi Fontana, M. (1997). Ciudadãos Modernos. Discurso è representação política. Campinas: Unicamp.

\section{Sobre el autor}

IgNACiO Rossi es Licenciado en Historia por la Universidad Nacional de Luján y maestrando en Historia Contemporánea por la Universidad Nacional de General Sarmiento. Investiga temas relacionados con la política económica argentina y forma parte del proyecto "Las interpretaciones heterodoxas en la historia económica argentina reciente: principales contribuciones y controversias" a cargo de la Dra. Forcinito Karina en el Área de Economía del Instituto del Desarrollo Humano. Correo Electrónico: ignacio.a.rossi@outlook.com. (iD https://orcid.org/0000-0003-3870-1630 


\title{
CUHSO
}

Fundada en 1984, la revista CUHSO es una de las publicaciones periódicas más antiguas en ciencias sociales y humanidades del sur de Chile. Con una periodicidad semestral, recibe todo el año trabajos inéditos de las distintas disciplinas de las ciencias sociales y las humanidades especializadas en el estudio y comprensión de la diversidad sociocultural, especialmente de las sociedades latinoamericanas y sus tensiones producto de la herencia colonial, la modernidad y la globalización. En este sentido, la revista valora tanto el rigor como la pluralidad teórica, epistemológica y metodológica de los trabajos.

\author{
EDITOR \\ Matthias Gloël \\ CoOrdinadora EDITORIAL \\ Claudia Campos Letelier \\ Corrector de ESTILO Y DiSEÑAdor \\ Ediciones Silsag \\ Traductor, CORRECTOR LENGUA INGLESA \\ Aurora Sambolin Santiago \\ SITIO WEB \\ cuhso.uct.cl \\ E-MAIL \\ cuhso@uct.cl
}

LICENCIA DE ESTE ARTÍCULO

Creative Commons Atribución Compartir Igual 4.0 Internacional 